\title{
VERITAS OBSERVATIONS OF THE MICROQUASAR CYGNUS X-3
}

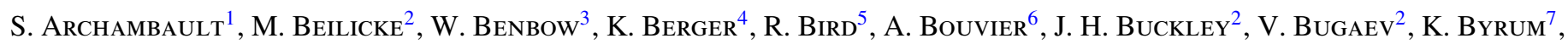
M. Cerrutt ${ }^{3}$, X. Chen $^{8,9}$, L. Ciupik ${ }^{10}$, M. P. Connolly ${ }^{11}$, W. Cui ${ }^{12,33}$, C. Duke ${ }^{13}$, J. Dumm ${ }^{14}$, M. Errando ${ }^{15}$, A. Falcone $^{16}$, S. Federici ${ }^{8,9}$, Q. Feng ${ }^{12}$, J. P. Finley ${ }^{12}$, L. Fortson ${ }^{14}$, A. Furniss ${ }^{6}$, N. Galante ${ }^{3}$, G. H. Gillanders ${ }^{11}$, S. Griffin ${ }^{1}$, S. T. Griffiths ${ }^{17}$, J. Grube ${ }^{10}$, G. GyuK ${ }^{10}$, D. Hanna ${ }^{1}$, J. Holder ${ }^{4}$, G. Hughes ${ }^{9}$, T. B. Humensky ${ }^{18}$, P. KaAret ${ }^{17}$, M. Kertzman ${ }^{19}$, Y. Khassen ${ }^{5}$, D. Kieda ${ }^{20}$, H. KrawCZYNSKI ${ }^{2}$, M. J. LanG ${ }^{11}$, A. S. Madhavan ${ }^{21}$, G. Maier ${ }^{9}$, P. Majumdar ${ }^{22,23}$, S. McArthur ${ }^{24}$, A. McCann ${ }^{25}$, P. Moriarty ${ }^{26}$, R. Mukherjee ${ }^{15}$, D. Nieto ${ }^{18}$, A. O'Faoláin de Bhróithe ${ }^{5}$, R. A. $\mathrm{ONG}^{22}$, A. N. Otte ${ }^{27}$, D. PAndel ${ }^{28}$, N. Park ${ }^{24}$, J. S. Perkins ${ }^{29}$, M. Pohl ${ }^{8,9}$, A. PopKow ${ }^{22}$, H. Prokoph ${ }^{9}$, J. Quinn ${ }^{5}$, K. RAGAN ${ }^{1}$, J. Rajotte ${ }^{1}$, L. C. Reyes ${ }^{30}$, P. T. Reynolds ${ }^{31}$, G. T. Richards ${ }^{27}$, E. Roache ${ }^{3}$, G. H. Sembroski ${ }^{12}$, F. Sheidaei ${ }^{20}$,

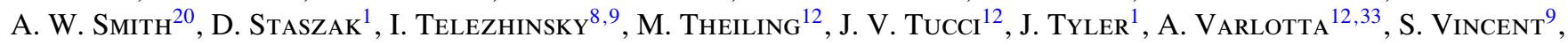
S. P. WaKely ${ }^{24}$, T. C. Weekes ${ }^{3}$, A. Weinstein ${ }^{21}$, D. A. Williams ${ }^{6}$, B. Zitzer ${ }^{7}$

(The VERITAS COLlaboration)

AND

M. L. MCCOLLOUGH ${ }^{32}$

(SMithsonian Astrophysical ObSERVATORy)

${ }^{1}$ Physics Department, McGill University, Montreal, QC H3A 2T8, Canada

2 Department of Physics, Washington University, St. Louis, MO 63130, USA

${ }^{3}$ Fred Lawrence Whipple Observatory, Harvard-Smithsonian Center for Astrophysics, Amado, AZ 85645, USA

${ }^{4}$ Department of Physics and Astronomy and the Bartol Research Institute, University of Delaware, Newark, DE 19716, USA

${ }^{5}$ School of Physics, University College Dublin, Belfield, Dublin 4, Ireland

${ }^{6}$ Santa Cruz Institute for Particle Physics and Department of Physics, University of California, Santa Cruz, CA 95064, USA

${ }^{7}$ Argonne National Laboratory, 9700 S. Cass Avenue, Argonne, IL 60439, USA

${ }^{8}$ Institute of Physics and Astronomy, University of Potsdam, D-14476 Potsdam-Golm, Germany

${ }^{9}$ DESY, Platanenallee 6, D-15738 Zeuthen, Germany

${ }^{10}$ Astronomy Department, Adler Planetarium and Astronomy Museum, Chicago, IL 60605, USA

${ }^{11}$ School of Physics, National University of Ireland Galway, University Road, Galway, Ireland

${ }^{12}$ Department of Physics, Purdue University, West Lafayette, IN 47907, USA; avarlott@purdue.edu, cui@purdue.edu

${ }^{13}$ Department of Physics, Grinnell College, Grinnell, IA 50112-1690, USA

${ }^{14}$ School of Physics and Astronomy, University of Minnesota, Minneapolis, MN 55455, USA

${ }^{15}$ Department of Physics and Astronomy, Barnard College, Columbia University, NY 10027, USA

${ }^{16}$ Department of Astronomy and Astrophysics, 525 Davey Lab, Pennsylvania State University, University Park, PA 16802, USA

${ }^{17}$ Department of Physics and Astronomy, University of Iowa, Van Allen Hall, Iowa City, IA 52242, USA

${ }^{18}$ Physics Department, Columbia University, New York, NY 10027, USA

${ }^{19}$ Department of Physics and Astronomy, DePauw University, Greencastle, IN 46135-0037, USA

${ }^{20}$ Department of Physics and Astronomy, University of Utah, Salt Lake City, UT 84112, USA

${ }^{21}$ Department of Physics and Astronomy, Iowa State University, Ames, IA 50011, USA

${ }^{22}$ Department of Physics and Astronomy, University of California, Los Angeles, CA 90095, USA

${ }^{23}$ Saha Institute of Nuclear Physics, Kolkata 700064, India

${ }^{24}$ Enrico Fermi Institute, University of Chicago, Chicago, IL 60637, USA

${ }^{25}$ Kavli Institute for Cosmological Physics, University of Chicago, Chicago, IL 60637, USA

${ }^{26}$ Department of Life and Physical Sciences, Galway-Mayo Institute of Technology, Dublin Road, Galway, Ireland

${ }^{27}$ School of Physics and Center for Relativistic Astrophysics, Georgia Institute of Technology, 837 State Street NW, Atlanta, GA 30332-0430, USA

${ }^{28}$ Department of Physics, Grand Valley State University, Allendale, MI 49401, USA

${ }^{29}$ NASA/Goddard Space Flight Center, Code 661, Greenbelt, MD 20771, USA

${ }^{30}$ Physics Department, California Polytechnic State University, San Luis Obispo, CA 94307, USA

${ }^{31}$ Department of Applied Physics and Instrumentation, Cork Institute of Technology, Bishopstown, Cork, Ireland

${ }^{32}$ Smithsonian Astrophysical Observatory, 60 Garden Street, Cambridge, MA 02138, USA

Received 2013 July 22; accepted 2013 October 28; published 2013 December 3

\section{ABSTRACT}

We report results from $\mathrm{TeV}$ gamma-ray observations of the microquasar Cygnus X-3. The observations were made with the Very Energetic Radiation Imaging Telescope Array System (VERITAS) over a time period from 2007 June 11 to 2011 November 28. VERITAS is most sensitive to gamma rays at energies between $85 \mathrm{GeV}$ and $30 \mathrm{TeV}$. The effective exposure time amounts to a total of about $44 \mathrm{hr}$, with the observations covering six distinct radio/ $\mathrm{X}$-ray states of the object. No significant $\mathrm{TeV}$ gamma-ray emission was detected in any of the states, nor with all observations combined. The lack of a positive signal, especially in the states where $\mathrm{GeV}$ gamma rays were detected, places constraints on $\mathrm{TeV}$ gamma-ray production in Cygnus $\mathrm{X}-3$. We discuss the implications of the results.

Key words: acceleration of particles - binaries: close - gamma rays: stars - X-rays: individual (Cygnus X-3)

Online-only material: color figures 


\section{INTRODUCTION}

Cygnus X-3 was among the first X-ray sources to be discovered in the early days of X-ray astronomy. It lies in the Galactic plane, at a distance between $7 \mathrm{kpc}$ and $10 \mathrm{kpc}$ (Predehl et al. 2000; Ling et al. 2009). It is a high-mass X-ray binary, with the companion star appearing to show the spectral characteristics of a Wolf-Rayet star (van Kerkwijk et al. 1996). The nature of the compact object is still being debated. Cygnus X-3 is known to produce intense radio flares, making it at times one of the brightest transient Galactic radio sources. The radio flares can last from a few days to several weeks. Relativistic jets have been seen during major flares $\left(S_{v}>10 \mathrm{Jy}\right.$ at $15 \mathrm{GHz}$; Mioduszewski et al. 2001; Miller-Jones et al. 2004), with an inclination to the line of sight of $\lesssim 14^{\circ}$. This makes Cygnus X-3 analogous to the extragalactic blazars, which constitute a major population of known TeV gamma-ray emitters. With an orbital period of only $4.8 \mathrm{hr}$, the compact object is thought to be enshrouded in the wind of the Wolf-Rayet star.

Cygnus X-3 has long been a prominent target for gammaray observations. Initially, there was contradictory evidence for gamma-ray emission at $\mathrm{GeV}$ energies: SAS-2 found a periodic signal (Lamb et al. 1977), while COS-B could not confirm it (Bennett et al. 1977). Then, numerous claims of detection of Cygnus X-3 were made in the early days of ground-based gamma-ray experiments, spanning the $\mathrm{TeV}$ to $\mathrm{PeV}$ energy range (see discussions by Weekes 1992 and Ong 1998). The claims were subsequently disputed by a critical analysis of the observations (Chardin \& Gerbier 1989). In subsequent years, Cygnus X-3 was observed with more sensitive groundbased instruments, including Whipple (O'Flaherty et al. 1992), CASA-MIA (Borione et al. 1997), HEGRA (Schilling et al. 2001), and MAGIC (Aleksić et al. 2010), but was not detected. At $\mathrm{GeV}$ energies, EGRET/CGRO found a gamma-ray source (2EG J2033+4112) that is consistent with the position of Cygnus $\mathrm{X}-3$ (although the position error circle is quite large), but with no evidence for orbital modulation (Mori et al. 1997). The source has now been detected at $\mathrm{GeV}$ energies, with high confidence, independently with AGILE (Tavani et al. 2009) and Fermi LAT (Abdo et al. 2009). Moreover, the orbital modulation of the gamma-ray emission has also been seen (Abdo et al. 2009).

In X-ray binaries, gamma rays may be produced by Compton upscattering of photons, from either the companion star or the accretion disk or both, by relativistic electrons accelerated in the jets of a stellar-mass black hole or in the shocked wind of a pulsar. Detailed models have been constructed for gamma-ray production and attenuation in the jets. In the case of Cygnus X-3, the close proximity $\left(R_{d} \approx 3 \times 10^{11} \mathrm{~cm}\right)$, high temperature $\left(T_{*} \sim 10^{5} \mathrm{~K}\right)$, and high luminosity $\left(L_{*} \sim\right.$ $10^{39} \mathrm{erg} \mathrm{s}^{-1}$ ) of the Wolf-Rayet star may result in the efficient Compton upscattering of stellar photons to produce gamma rays, as well as in the attenuation of the gamma rays via $\gamma \gamma$ pair production (Bednarek 2010). Whether Cygnus $\mathrm{X}-3$ appears as a $\mathrm{TeV}$ gamma-ray emitter would depend on the competition between the production and attenuation processes. Theoretically, certain circumstances would favor $\mathrm{TeV}$ emission. These typically involve emitting regions at large perpendicular distances $\left(H \gtrsim 10 R_{d}\right)$ from the orbital plane and orbital phases around the inferior conjunction (Bednarek 2010). At lower $(\mathrm{GeV})$ energies, the attenuation optical depth is much reduced, so the observed orbital modulation may be mainly asso-

\footnotetext{
33 Authors to whom any correspondence should be addressed.
}

ciated with the production process (Dubus et al. 2010; Bednarek 2010). Alternatively, gamma rays may also be produced by the decay of $\pi^{0}$ particles, which result from the $p p$ collisions between the relativistic protons in the jets and the cold protons of the dense anisotropic stellar wind of the Wolf-Rayet star (Romero et al. 2003).

Cygnus X-3 is a persistent radio source. Its radio flux may vary by four orders of magnitude. Based on the long-term monitoring of the source with the Green Bank Interferometer, four radio states were identified (Waltman et al. 1994): quiescent state (60-140 mJy), minor flaring state $(\lesssim 1 \mathrm{Jy})$, quenched state $(\lesssim 30 \mathrm{mJy})$, and intermediate/major flaring state $(>1 \mathrm{Jy})$. The major flaring state seems to follow the quenched state. The radio emission was subsequently found to be correlated with the hard X-ray emission (McCollough et al. 1999). The correlation is complex and varies with the state that the source is in: it is negative (anti-correlation) in the quiescent state but turns positive in the major flaring and quenched states. No apparent correlation has been observed in the minor flaring state. The radio emission is also correlated with the soft X-ray emission in certain states (Hjalmarsdotter et al. 2008). This is expected because it is known that the soft and hard X-ray fluxes of Cygnus $\mathrm{X}-3$ are generally (but not always) anti-correlated (Choudhury et al. 2002; Hjalmarsdotter et al. 2008). Based on the correlated radio/X-ray properties of the source, Szostek et al. (2008) refined and expanded the definitions of the states. The new radio/X-ray states are now referred to as the quiescent, minorflaring, suppressed, quenched, major-flaring, and post-flaring states.

The AGILE and Fermi-LAT observations have shown that the gamma-ray emission from Cygnus X-3 is not steady but episodic. A careful examination of the gamma-ray activities of the source has revealed that gamma-ray production appears to be associated with transitions into or out of the radio quenched state (Koljonen et al. 2010). During a transition, the X-ray spectrum of the source becomes dominated by a soft X-ray component (with only a weak power-law component) as its radio flux goes down. For this reason, these time periods are now also referred to as the hypersoft state (Koljonen et al. 2010). As such, the line between the hypersoft state and quenched state is not always very clear in practice. Observationally, the hypersoft state is associated with major radio flares and, sometimes, the formation of jets (Koljonen et al. 2010). The latter might be the site of gamma-ray production. This signifies the importance of the hypersoft state to our understanding of Cygnus X-3 as a gammaray emitter. Unfortunately, the hypersoft state is very short in duration (lasting for $\lesssim 4-5$ days), compared with other states, so it is often challenging to catch it with sensitive instruments of small field of view (FOV).

In this work, we carried out a systematic search for gamma rays from Cygnus X-3 at TeV energies with the Very Energetic Radiation Imaging Telescope Array System (VERITAS). The availability of the contemporaneous radio/X-ray observations of the source made it possible to extend the search to individual radio/X-ray states, particularly to the hypersoft state.

\section{VERITAS OBSERVATIONS}

VERITAS is a ground-based gamma-ray telescope array located at the Fred Lawrence Whipple Observatory in southern Arizona in the United States. It consists of four $12 \mathrm{~m}$ imaging atmospheric Cerenkov telescopes, each with 499-pixel cameras, designed to detect the faint flashes of Cerenkov light from air showers initiated in the atmosphere by $\mathrm{TeV}$ gamma rays or 
cosmic rays. VERITAS can detect gamma rays in the energy range from $85 \mathrm{GeV}$ to $30 \mathrm{TeV}$, with a maximum effective area of approximately $10^{5} \mathrm{~m}^{2}$. The energy resolution is about $15 \%-25 \%$. At $1 \mathrm{TeV}$, the angular resolution is better than 0.1 for an individual gamma event ( $68 \%$ containment level). The pointing accuracy of VERITAS is $<50^{\prime \prime}$. VERITAS has an FOV of about 3.5 in angular diameter. In the summer of 2009, one of the four telescopes of the array was relocated to a different position, increasing the overall sensitivity of the array by about $30 \%$. After the relocation, VERITAS is capable of detecting sources at the flux level of $1 \%$ of the Crab Nebula with a $\sim 25 \mathrm{hr}$ exposure (Holder et al. 2011).

For this work, we used data from observations conducted between 2007 June 11 and 2011 November 28. The observations were conducted under varying weather and other conditions. The design of VERITAS also allows observations to be conducted under partial moonlight. To mitigate the night sky background fluctuations caused by the moonlight, the triggering threshold is increased in the camera photomultiplier tubes, which leads to a higher energy threshold. The triggering threshold in the photomultiplier tubes sets the first step in event discrimination in the telescope array. We carefully examined the data and included all of the observations that we believe can lead to reliable results. The total exposure time amounts to about $44 \mathrm{hr}$. More detailed information on the observations is shown in Table 1.

The reduction of VERITAS data consists of multiple steps, including rejection of substandard data, flat fielding, pedestal subtraction, pulse integration, image cleaning, parameterization of events, stereo reconstruction of shower direction and impact parameter, and gamma-ray/cosmic-ray separation. Briefly, the data from each participating telescope are first filtered for bad weather or issues with data acquisition and are then charge integrated, pedestal subtracted, and gain corrected. Each resulting image is cleaned and characterized to derive the moments of the light distributions (Hillas 1985). The images of the same air shower from all participating telescopes are used to reconstruct the direction and impact parameter of the shower (see, e.g., Krawczynski et al. 2006). This step requires characterizable images from three or more telescopes. In addition, to separate the gamma-ray events from the cosmic-ray events, we applied selection criteria (based on the energy and geometry of the events) to the events that survived the previous steps. The post-selection energy threshold is about $220 \mathrm{GeV}$ at a $10^{\circ}$ zenith angle and $450 \mathrm{GeV}$ at a $40^{\circ}$ zenith angle, which correspond approximately to the lowest and highest zenith angle of our data set, respectively. More details about VERITAS, the calibration procedure, and the analysis technique can be found in Acciari et al. (2008).

The VERITAS observation set was the product of different observation modes: it was composed of wobble-mode data on Cygnus X-3, wobble-mode data taken on the TeV gammaray source $\mathrm{TeV} \mathrm{J} 2032+4130$ (Aharonian et al. 2005), which is $\sim 30^{\prime}$ from Cygnus X-3, and data from tracking mode on the mid-point position between Cygnus X-3 and TeV J2032+4130. In wobble mode, the telescopes are pointed such that the source is always located at a fixed offset (0.5), alternately to the north, south, east, and west of the camera center, for an unbiased estimation of the FOV background of the source region. In tracking mode, the telescopes were pointed alternatively to the east and west of the mid-point position between Cygnus X-3 and TeV J2032+4130. Due to the mixture of different observing modes, the data analysis used the ring background model (Berge et al. 2007). Briefly, the background estimate is derived for a trial source position from an annulus around the source region, which is dependent on the selection criteria. Due to the different offsets of the ring points with respect to the camera center as compared to the source position, a relative event rate, or acceptance, correction needs to be applied to normalize the background rate. Any gamma-ray source in the FOV needs to be excluded from the background estimation as well. In our case, we excluded the pixels pointing at bright stars (with $B$ magnitude less than 6) from the background regions. The nearby known $\mathrm{TeV}$ gamma-ray source, $\mathrm{TeV} \mathrm{J} 2032+4130$, was removed from subsequent analyses, to avoid incorrect estimation of the source and background rates of Cygnus X-3 in the analysis.

The data analysis on Cygnus X-3 was performed with selection-criteria parameters based on the energy and geometry configuration of the gamma-ray initiated air showers and modeled on the Crab Nebula. The selection criteria are optimized for a putative source with either a soft $(6.6 \% \mathrm{Crab}$ at $200 \mathrm{GeV}$, spectral index: -4$)$, medium $(2 \% \mathrm{Crab}$ at $400 \mathrm{GeV}$, spectral index: -2.4$)$, or hard $(2 \% \mathrm{Crab}$ at $1 \mathrm{TeV}$, spectral index: -2.0) spectral index. The selection-criteria parameters tend to be looser for softer sources than for harder ones, to allow less event selection restrictions in the analysis. The acceptance correction was consequently generated over the whole data set for soft, medium, and hard selection criteria and then applied to partial data sets (e.g., for individual states). For data taken with the initial VERITAS telescope array configuration (prior to 2009 August) where telescope 1 (T1) and telescope 4 (T4) were in proximity to one another, all $\mathrm{T} 1$ and $\mathrm{T} 4$ simultaneous events were removed from analysis if no other telescope (T2 or T3) was triggered.

\section{SUPPORTING MULTI-WAVELENGTH OBSERVATIONS}

To gain a broadband perspective, we also examined data from observations at longer wavelengths. Particularly relevant to this work are contemporaneous gamma-ray observations of Cygnus $\mathrm{X}-3$ at GeV energies made with the Large Area Telescope (LAT) aboard the Fermi Gamma-Ray Space Telescope satellite (Atwood et al. 2009), as well as contemporaneous X-ray and radio observations, which make it possible to characterize the radio/X-ray states of the source.

\subsection{Fermi-LAT Observations}

In the default survey mode, the LAT scans the sky continuously and covers the whole sky once about every three hours. It is sensitive to gamma rays in the nominal energy range of $0.02-300 \mathrm{GeV}$. Its on-axis effective area is between 8000 and $9000 \mathrm{~cm}^{2}$ for energies $\gtrsim 1 \mathrm{GeV}$. The LAT has a very large FOV $(\sim 2.4 \mathrm{sr})$ and has an angular resolution of better than 0.1 at $1 \mathrm{GeV}$ (for $68 \%$ containment). For this work, we used the LAT data taken from 2008 August 5 to 2012 March 13.

The LAT data were processed with the Fermi Science Tools (v9r23p1), following the recommendations on event selection from the Fermi Science Support Center. ${ }^{34}$ Briefly, the events that have the highest probability of being gamma rays were selected by means of the Pass 7 V6 (P7_V6) source class event selection cut with the gtselect tool. In order to minimize contamination from Earth albedo photons, the time periods when Cygnus X-3 was observed at zenith angles greater than $100^{\circ}$ were eliminated from further analysis. The energy range was also limited from 0.1 to $100 \mathrm{GeV}$.

\footnotetext{
34 http://fermi.gsfc.nasa.gov/ssc/data/analysis/scitools/
} 
Table 1

Summary of VERITAS Observations

\begin{tabular}{|c|c|c|c|c|c|}
\hline MJD & $\begin{array}{l}\text { Calendar } \\
\text { Date }\end{array}$ & $\begin{array}{l}\text { X-Ray } \\
\text { State }\end{array}$ & $\begin{array}{l}\text { Observing Time } \\
\text { (min) }\end{array}$ & $\begin{array}{c}\text { Elevation Range } \\
\text { (deg) }\end{array}$ & $N_{\text {tel }}$ \\
\hline 54,262 & 2007 Jun 11 & Minor flaring & 20 & $65-69$ & 3 \\
\hline 54,263 & 2007 Jun 12 & Minor flaring & 40 & $70-77$ & 3 \\
\hline 54,264 & 2007 Jun 13 & Minor flaring & 119.5 & $62-80$ & 3 \\
\hline 54,265 & 2007 Jun 14 & Minor flaring & 80 & $72-80$ & 3 \\
\hline 54,266 & 2007 Jun 15 & Minor flaring & 40 & $72-78$ & 3 \\
\hline 54,626 & 2008 Jun 9 & Quenched & 40 & $76-80$ & 4 \\
\hline 54,627 & 2008 Jun 10 & Quenched & 40 & $76-80$ & 4 \\
\hline 54,628 & 2008 Jun 11 & Quenched & 20 & $80-81$ & 4 \\
\hline 54,731 & 2008 Sep 22 & Suppressed & 20 & $76-78$ & 4 \\
\hline 54,774 & 2008 Nov 4 & Quenched & 20 & $59-63$ & 4 \\
\hline 54,786 & 2008 Nov 16 & Major flaring & 60 & $59-72$ & 4 \\
\hline 54,789 & 2008 Nov 19 & Quenched & 60 & $64-68$ & 4 \\
\hline 54,794 & 2008 Nov 24 & Quenched & 40 & $54-60$ & 4 \\
\hline 54,800 & 2008 Nov 30 & Quenched & 20 & $54-58$ & 3 \\
\hline 54,804 & 2008 Dec 4 & Hypersoft $^{\mathrm{a}}$ & 20 & $53-56$ & 4 \\
\hline 55,126 & 2009 Oct 22 & Quiescent & 20 & $74-76$ & 4 \\
\hline 55,127 & 2009 Oct 23 & Quiescent & 36 & $67-74$ & 4 \\
\hline 55,128 & 2009 Oct 24 & Quiescent & 77 & $59-79$ & 4 \\
\hline 55,129 & 2009 Oct 25 & Quiescent & 40 & $65-74$ & 4 \\
\hline 55,155 & 2009 Nov 20 & Quiescent & 20 & $59-62$ & 3 \\
\hline 55,156 & 2009 Nov 21 & Quiescent & 40 & $56-64$ & 4 \\
\hline 55,157 & 2009 Nov 22 & Quiescent & 20 & $56-59$ & 4 \\
\hline 55,158 & 2009 Nov 23 & Quiescent & 16 & $64-67$ & 4 \\
\hline 55,382 & $2010 \mathrm{Jul} 5$ & Minor flaring & 20 & $72-76$ & 4 \\
\hline 55,384 & 2010 Jul 7 & Minor flaring & 4 & $80-80$ & 4 \\
\hline 55,481 & 2010 Oct 12 & Quiescent & 40 & $75-80$ & 4 \\
\hline 55,482 & 2010 Oct 13 & Quiescent & 40 & $69-77$ & 4 \\
\hline 55,648 & 2011 Mar 28 & Major flaring & 20 & $42-46$ & 4 \\
\hline 55,649 & 2011 Mar 29 & Major flaring & 20 & $42-46$ & 3 \\
\hline 55,650 & 2011 Mar 30 & Major flaring & 20 & $43-48$ & 4 \\
\hline 55,651 & 2011 Mar 31 & Major flaring & 28 & $45-51$ & 3 \\
\hline 55,652 & 2011 Apr 1 & Major flaring & 20 & $42-46$ & 3 \\
\hline 55,653 & 2011 Apr 2 & Major flaring & 20 & $42-46$ & 4 \\
\hline 55,654 & 2011 Apr 3 & Major flaring & 15 & $48-50$ & 4 \\
\hline 55,655 & 2011 Apr 4 & Major flaring & 20 & $48-50$ & 4 \\
\hline 55,656 & 2011 Apr 5 & Major flaring & 20 & $48-52$ & 4 \\
\hline 55,658 & 2011 Apr 7 & Minor flaring & 8 & $52-53$ & 3 \\
\hline 55,659 & 2011 Apr 8 & Minor flaring & 23 & $50-53$ & 4 \\
\hline 55,662 & 2011 Apr 11 & Minor flaring & 6 & $55-56$ & 3 \\
\hline 55,707 & 2011 May 26 & Minor flaring & 20 & $72-74$ & 4 \\
\hline 55,708 & 2011 May 27 & Minor flaring & 20 & $60-64$ & 4 \\
\hline 55,709 & 2011 May 28 & Minor flaring & 96 & $65-80$ & 4 \\
\hline 55,710 & 2011 May 29 & Minor flaring & 52 & $70-77$ & $3 / 4^{b}$ \\
\hline 55,713 & 2011 Jun 1 & Minor flaring & 20 & $59-62$ & 4 \\
\hline 55,715 & 2011 Jun 3 & Minor flaring & 40 & $77-80$ & 4 \\
\hline 55,716 & 2011 Jun 4 & Minor flaring & 20 & $70-74$ & 4 \\
\hline 55,717 & 2011 Jun 5 & Minor flaring & 20 & $79-81$ & 4 \\
\hline 55,720 & 2011 Jun 8 & Minor flaring & 20 & $76-78$ & 4 \\
\hline 55,721 & 2011 Jun 9 & Minor flaring & 10 & $74-75$ & 4 \\
\hline 55,733 & 2011 Jun 21 & Minor flaring & 14 & $45-48$ & 4 \\
\hline 55,734 & 2011 Jun 22 & Minor flaring & 10 & $64-68$ & 4 \\
\hline 55,735 & 2011 Jun 23 & Minor flaring & 46 & $59-69$ & 4 \\
\hline 55,736 & 2011 Jun 24 & Minor flaring & 84 & $74-80$ & 4 \\
\hline 55,737 & 2011 Jun 25 & Minor flaring & 40 & $69-73$ & 4 \\
\hline 55,738 & 2011 Jun 26 & Quiescent & 59.5 & $74-80$ & 4 \\
\hline 55,739 & 2011 Jun 27 & Quiescent & 95 & $71-80$ & 4 \\
\hline 55,740 & 2011 Jun 28 & Quiescent & 30 & $76-80$ & 3 \\
\hline 55,743 & 2011 Jul 1 & Quiescent & 20 & $76-80$ & 4 \\
\hline 55,744 & 2011 Jul 2 & Quiescent & 20 & $76-78$ & 4 \\
\hline 55,830 & 2011 Sep 26 & Quiescent & 80 & $78-80$ & $3 / 4^{b}$ \\
\hline 55,833 & 2011 Sep 29 & Quiescent & 48 & $74-80$ & 4 \\
\hline 55,834 & 2011 Sep 30 & Quiescent & 52 & $64-79$ & $3 / 4^{b}$ \\
\hline 55,835 & 2011 Oct 1 & Quiescent & 43.5 & $66-75$ & 4 \\
\hline 55,850 & 2011 Oct 16 & Quiescent & 28 & $74-80$ & 4 \\
\hline
\end{tabular}


Table 1

(Continued)

\begin{tabular}{lclccc}
\hline \hline MJD & $\begin{array}{c}\text { Calendar } \\
\text { Date }\end{array}$ & $\begin{array}{c}\text { X-Ray } \\
\text { State }\end{array}$ & $\begin{array}{c}\text { Observing Time } \\
(\text { min })\end{array}$ & $\begin{array}{c}\text { Elevation Range } \\
(\text { deg })\end{array}$ & $N_{\text {tel }}$ \\
\hline 55,851 & 2011 Oct 17 & Quiescent & 80 & $72-80$ & 4 \\
55,852 & 2011 Oct 18 & Quiescent & 71 & $68-80$ & 4 \\
55,853 & 2011 Oct 19 & Quiescent & 51 & $70-79$ & 4 \\
55,854 & 2011 Oct 20 & Quiescent & 111 & $70-79$ & 4 \\
55,855 & 2011 Oct 21 & Quiescent & 20 & $58-60$ & 4 \\
55,858 & 2011 Oct 24 & Quiescent & 20 & $59-61$ & 4 \\
55,860 & 2011 Oct 26 & Quiescent & 20 & $58-61$ & 4 \\
55,861 & 2011 Oct 27 & Quiescent & 17 & $70-74$ & 4 \\
55,862 & 2011 Oct 28 & Quiescent & 72 & $59-80$ & 4 \\
55,863 & 2011 Oct 29 & Quiescent & 35 & $74-80$ & 4 \\
55,864 & 2011 Oct 30 & Quiescent & 15 & $66-76$ & 4 \\
55,865 & 2011 Oct 31 & Quiescent & 40 & $60-68$ & 4 \\
55,888 & 2011 Nov 23 & Quiescent & 36 & $61-64$ & 4 \\
55,891 & 2011 Nov 26 & Quiescent & 20 & $56-64$ & 4 \\
55,892 & 2011 Nov 27 & Quiescent & 40 & $52-56$ & 4 \\
55,893 & 2011 Nov 28 & Quiescent & 20 & & 4 \\
\hline
\end{tabular}

Notes. The column $N_{\text {tel }}$ shows the number of working telescopes.

a The hypersoft state consists of the data run contained within the quenched state. See text.

${ }^{\mathrm{b}}$ One telescope was taken out of the operation during the run.

For background modeling, we included all of the sources in the Fermi Large Area Telescope Second Source (2FGL) Catalog (Nolan et al. 2012) that are in the vicinity of Cygnus $\mathrm{X}-3$. To account for possible intrinsic variability of the sources, we allowed the spectral parameters of the sources in a $5^{\circ}$ radius region of interest (RoI) to vary in an unbinned maximum likelihood analysis. On the other hand, we froze the spectral parameters of the sources that are outside of the RoI but within a $22^{\circ}$ radius source region at the $2 \mathrm{FGL}$ values. To minimize contamination from a bright nearby pulsar, PSR J2032+4127 (about 30" away from Cygnus X-3), following Corbel et al. (2012), we excluded the times of its peak-pulse emission, based on the pulsar ephemeris ${ }^{35}$ (Ray et al. 2011). As for the Galactic and extragalactic diffuse gamma-ray backgrounds, we adopted the most recent models (gal_2yearp7v6_v0.fits and iso_p7v6source.txt). We also modeled the emission from the Cygnus Loop region with a template that is provided in the LAT Catalog Data Products. The instrument response function (IRF) used in this work is IRF P7SOURCE_V6.

We derived, from background modeling, the best-fit spectral parameters of the sources in the RoI. We then fixed the parameters for all other sources, as well as the spectral index of Cygnus X-3, and performed another unbinned maximum likelihood analysis, to produce a light curve of Cygnus X-3 over the time period of interest. The statistical significance of each measurement is quantified by a maximum likelihood test statistic (TS; Mattox et al. 1996), which corresponds roughly to $\sqrt{\mathrm{TS}} \sigma$ in Gaussian statistics.

\subsection{X-Ray and Radio Observations}

Contemporaneous X-ray coverages of Cygnus X-3 were provided by the All-sky Monitor (ASM) aboard the RXTE satellite (Levine et al. 1996), the Burst Alert Monitor (BAT) aboard the Swift satellite (Barthelmy et al. 2005), and the Monitor of All-sky X-ray Image (MAXI) aboard the International Space

\footnotetext{
35 The pulsar ephemeris for PSR J2032+4127 is available at http://www.slac.stanford.edu/ abdo/LATPulsarTimingModels/Latest/ J2032+4127/J2032+4127_latest.par.
}

Station (Matsuoka et al. 2009). The ASM and MAXI cover soft $\mathrm{X}$-ray bands of $1.5-12 \mathrm{keV}$ and 2-20 keV, respectively, while the BAT covers the hard X-ray band of $15-50 \mathrm{keV}$. For this work, we chose to use the ASM and MAXI data in a narrower (soft) band, to achieve a more accurate characterization of the states (Szostek et al. 2008). We weighted the measured count rates or fluxes of Cygnus X-3 (by $1 / \sigma^{2}$ ), which are made publicly available by the instrument teams and, if necessary, rebinned them to produce daily-averaged light curves.

At radio wavelengths, Cygnus X-3 is monitored regularly with the Arcminute Microkelvin Imager-Large Array (AMILA) ${ }^{36}$ at the Mullard Radio Astronomy Observatory in the UK. The AMI-LA consists of eight $12.8 \mathrm{~m}$ Cassegrain antennas in a two-dimensional array, with a baseline of $\sim 120 \mathrm{~m}$ (Zwart et al. 2008). It operates in six frequency bands covering the range of 13.9-18.2 GHz. Here, we used the data taken from 2008 May 26 to 2011 December 31 . Note that no data were taken between 2006 June 19 and 2008 May 26, due to the major upgrade of the Ryle Radio Telescope to the AMI-LA. The weighted, dailyaveraged light curve was used for this work.

\section{RESULTS}

\subsection{Blind Searches for TeV Gamma Rays}

Using the full VERITAS data set, we found no significant $(>5 \sigma)$ excess of $\mathrm{TeV}$ gamma rays from Cygnus $\mathrm{X}-3$ with the soft, medium, and hard data cuts. The significance was calculated with the modified Equation (17) of Li \& Ma (1983), which is generalized for data sets with different source and background regions (Aharonian et al. 2004). The results are summarized in Table 2.

To derive a flux upper limit for each observing run, we calculated the total counts in the source region $N_{\text {on }}$, total counts in the background region $N_{\text {off }}$, and a scale factor $\alpha$, which is defined as the ratio of the areas of the (geometrical or parameter) regions from which source and background counts are derived. The scale factor may be different for different cuts.

\footnotetext{
36 http://www.mrao.cam.ac.uk/ guy/cx3/
} 
Table 2

Results from Gamma-Ray Searches

\begin{tabular}{|c|c|c|c|c|c|c|c|c|c|}
\hline $\begin{array}{l}\text { Spectral } \\
\text { State }\end{array}$ & $\begin{array}{c}\text { Exposure } \\
\text { Time } \\
(\mathrm{hr})\end{array}$ & $\begin{array}{l}\text { Elevation } \\
\text { Range }\end{array}$ & $\begin{array}{c}\text { On } \\
\text { Events } \\
N_{\text {on }} \\
\end{array}$ & $\begin{array}{c}\text { Off } \\
\text { Events } \\
N_{\text {off }} \\
\end{array}$ & $\alpha_{\text {eff }}$ & $\begin{array}{c}\text { Excess } \\
\text { Events } \\
N_{e x} \\
\end{array}$ & $\begin{array}{l}\text { Significance } \\
\qquad(\sigma)\end{array}$ & $\begin{array}{c}\text { Energy } \\
\text { Threshold } \\
(\mathrm{GeV})\end{array}$ & $\begin{array}{l}\text { Flux Upper Limit } \\
\left(10^{-12} \mathrm{~cm}^{-2} \mathrm{~s}^{-1}\right)\end{array}$ \\
\hline \multicolumn{10}{|c|}{ Soft Cuts } \\
\hline All & 44.70 & $42^{\circ}-81^{\mathrm{o}}$ & 17509 & 125799 & 0.136 & 400.3 & 0.6 & 182 & 5.0 \\
\hline Quiescent & 23.04 & $52^{\circ}-80^{\circ}$ & 9046 & 65596 & 0.136 & 124.9 & 0.3 & 182 & 4.6 \\
\hline Minor flaring & 13.68 & $45^{\circ}-81^{\mathrm{o}}$ & 4032 & 28865 & 0.138 & 48.6 & 0.6 & 200 & 6.1 \\
\hline Suppressed & 0.30 & $76^{\circ}-78^{\circ}$ & 162 & 1069 & 0.156 & -4.8 & -0.4 & 200 & 64.6 \\
\hline Quenched & 4.24 & $54^{\circ}-81^{\circ}$ & 2410 & 16923 & 0.142 & 6.9 & 0.1 & 200 & 20.5 \\
\hline Hypersoft $^{\mathrm{a}}$ & 0.30 & $53^{\circ}-63^{\circ}$ & 180 & 1360 & 0.142 & -13.1 & -0.9 & 316 & 29.0 \\
\hline Major flaring & 3.44 & $42^{\circ}-72^{\circ}$ & 1859 & 13344 & 0.138 & 17.5 & 0.4 & 316 & 10.9 \\
\hline \multicolumn{10}{|c|}{ Medium Cuts } \\
\hline All & 44.70 & $42^{\circ}-81^{\circ}$ & 1200 & 26176 & 0.046 & -4.1 & -0.1 & 263 & 0.7 \\
\hline Quiescent & 23.04 & $52^{\circ}-80^{\circ}$ & 654 & 15268 & 0.046 & -48.3 & -1.7 & 263 & 0.5 \\
\hline Minor flaring & 13.68 & $45^{\circ}-81^{\circ}$ & 343 & 6813 & 0.046 & 29.6 & 1.5 & 288 & 2.1 \\
\hline Suppressed & 0.30 & $76^{\circ}-78^{\circ}$ & 11 & 94 & 0.047 & 6.6 & 2.5 & 288 & 41.8 \\
\hline Quenched & 4.24 & $54^{\circ}-81^{\circ}$ & 96 & 2097 & 0.047 & -2.6 & -0.1 & 347 & 2.5 \\
\hline Hypersoft $^{\mathrm{a}}$ & 0.30 & $53^{\circ}-63^{\circ}$ & 8 & 205 & 0.045 & -1.2 & -0.4 & 457 & 9.0 \\
\hline Major flaring & 3.44 & $42^{\circ}-72^{\circ}$ & 96 & 1904 & 0.047 & 6.5 & 0.7 & 550 & 2.2 \\
\hline \multicolumn{10}{|c|}{ Hard Cuts } \\
\hline All & 44.70 & $42^{\circ}-81^{\circ}$ & 145 & 3305 & 0.045 & -3.7 & -0.2 & 603 & 0.2 \\
\hline Quiescent & 23.04 & $52^{\circ}-80^{\circ}$ & 68 & 1936 & 0.045 & -19.1 & -2.0 & 603 & 0.1 \\
\hline Minor flaring & 13.68 & $45^{\circ}-81^{\circ}$ & 46 & 831 & 0.046 & 7.8 & 1.2 & 603 & 0.6 \\
\hline Suppressed & 0.30 & $76^{\circ}-78^{\circ}$ & 1 & 14 & 0.045 & 0.4 & 0.4 & 603 & 10.2 \\
\hline Quenched & 4.24 & $54^{\circ}-81^{\circ}$ & 13 & 281 & 0.046 & 0.1 & 0.0 & 871 & 0.9 \\
\hline Hypersoft $^{\mathrm{a}}$ & 0.30 & $53^{\circ}-63^{\circ}$ & 3 & 25 & 0.042 & 2.0 & 1.5 & 871 & 9.2 \\
\hline Major flaring & 3.44 & $42^{\circ}-72^{\circ}$ & 17 & 244 & 0.047 & 5.5 & 1.5 & 955 & 1.2 \\
\hline
\end{tabular}


background calculation (see Section 4).

a The hypersoft state consists of the data run from 2008/12/04 (MJD 54804) and is a data run subset contained within the quenched state.

It may also vary from run to run, because, for instance, a bright star or known gamma source may need to be excluded from the background region in certain wobble configurations. For the analyses of multiple data runs, individual $\alpha$ values were weighted by corresponding background counts and averaged to produce an effective $\alpha_{\text {eff }}$ for the runs. To account for varying zenith angle conditions, an average effective area $A_{\text {eff }}$ was constructed from individual effective areas for the runs. The flux upper limit was then derived from total $N_{\text {on }}$, total $N_{\text {off }}, \alpha_{\text {eff }}$, $A_{\text {eff }}$, and total effective exposure time, with the method of Rolke et al. (2005).

Table 2 shows the $95 \%$ confidence level (C.L.) integral flux upper limits derived with the full VERITAS data set. We chose as the lower limit for flux integration the energy threshold, which is defined as the energy at which the differential rate of gamma-ray detection as a function of energy reaches its maximum. Different data cuts lead to different energy thresholds (also shown in the table). We should point out that we did not include systematic uncertainties in this or subsequent analyses.

\subsubsection{Search for Episodic Emission}

We also conducted a blind search for episodic TeV gamma-ray emission from Cygnus X-3. In this case, the VERITAS data runs were grouped on a night-by-night basis. As before, we selected events with the soft, medium, and hard cuts, respectively, and followed the same procedure to reduce and analyze the data. Figure 1 shows the distribution of the significance of excess for each set of cuts separately. The distributions are consistent with no significant $\mathrm{TeV}$ gamma-ray signal from Cygnus $\mathrm{X}-3$ (with the $99 \%$ C.L. integral flux upper limits shown in the top panel of Figure 4 for individual nights).

\subsubsection{Search for Orbital Modulation}

Considering that gamma-ray production could be concentrated in certain parts of the binary orbit, we folded the data from all observing runs into 10 phase bins, using the ephemeris of Zdziarski et al. (2012). When a run spans multiple phase bins, we took care in dividing it so that the events fall in the correct bins. Again, we followed the same procedure to reduce and analyze the runs (or sub-runs) for each phase bin. We found no significant excess over the entire orbit. The 95\% C.L. integral flux upper limits (derived with the medium cuts) are shown in Figure 2.

\subsubsection{Spectral Constraints}

To place constraints on the gamma-ray spectrum of Cygnus $\mathrm{X}-3$ at TeV energies, we also analyzed the data for selected energy ranges. The 95\% C.L. integral flux upper limits are given in Table 3, and the corresponding differential flux upper limits are shown in Figure 3. We adopted logarithmic energy binning $(\Delta E / E \sim 30 \%)$ for this analysis. The bins are coarser than the energy resolution of VERITAS but are sufficiently small to minimize any spectral dependence of the results. Such dependence may arise from the fact that the effective area is constructed, via Monte-Carlo simulations, with an assumed input spectrum (which, in this case, has a photon index of -2.4) 

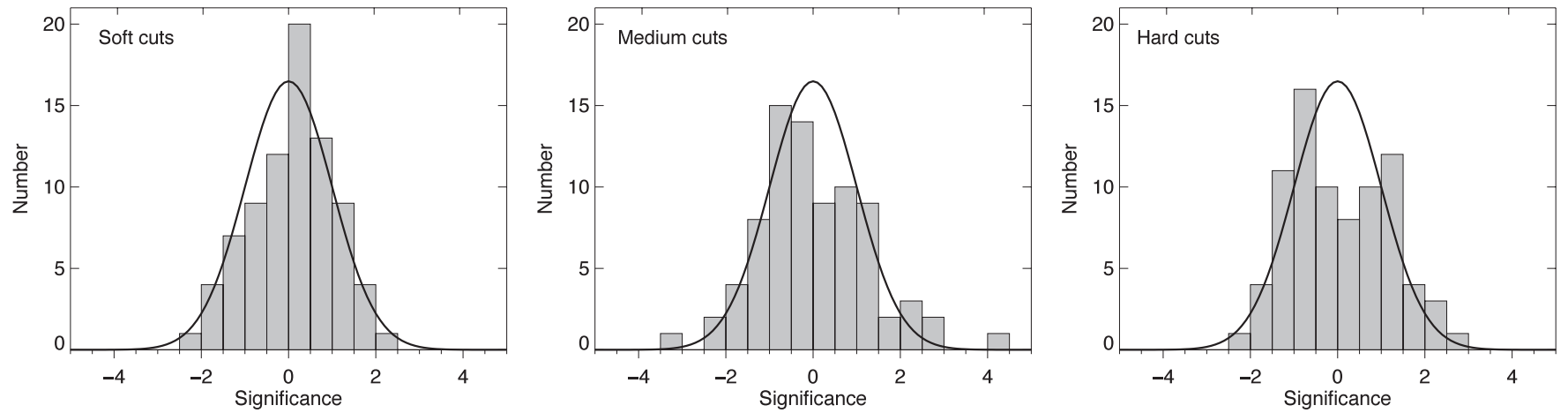

Figure 1. Gaussian significance (in units of $\sigma$ ) distributions for VERITAS nightly searches. The results from different data cuts are shown separately. The Gaussian functions with mean zero and $\sigma$ one are shown in solid lines.

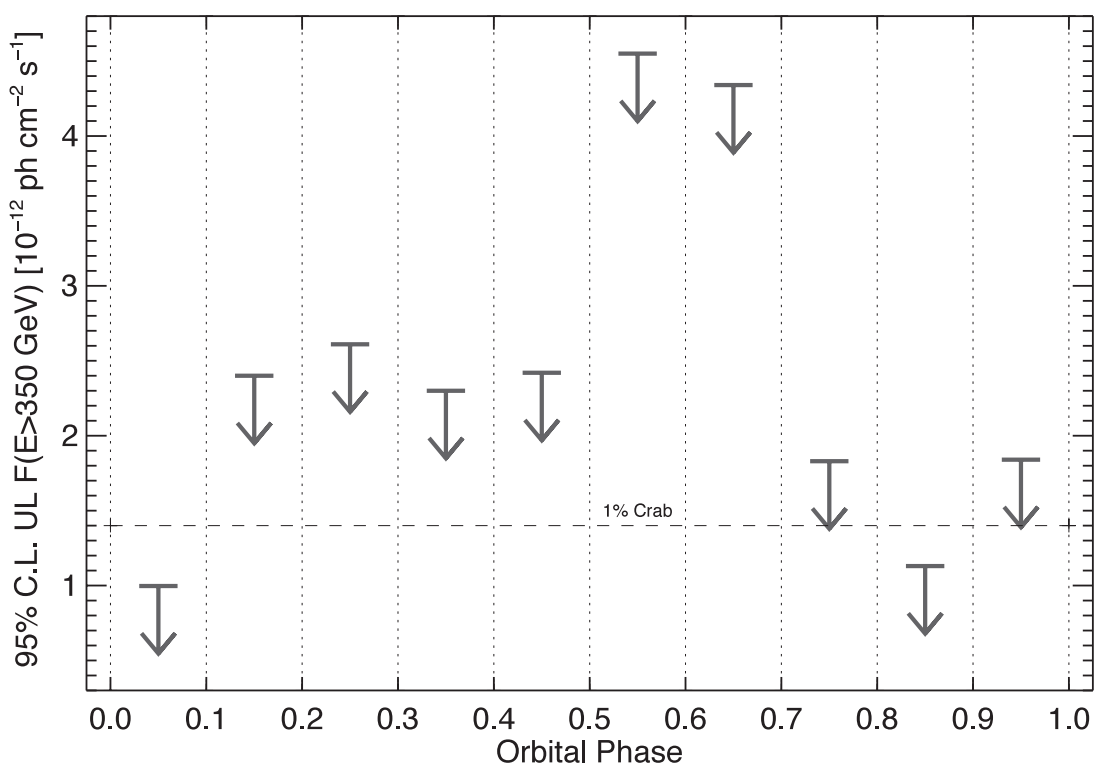

Figure 2. VERITAS phase-folded 95\% C.L. integral ( $E>350 \mathrm{GeV}$ ) flux upper limits of Cygnus X-3. For reference, the level of $1 \%$ Crab is indicated (in dashed line).

Table 3

Flux Upper Limits for Selected Energy Ranges

\begin{tabular}{lcccccc}
\hline \hline $\begin{array}{l}\text { Energy Range } \\
(\mathrm{TeV})\end{array}$ & $\begin{array}{c}\text { On } \\
\text { Events } \\
N_{\text {on }}\end{array}$ & $\begin{array}{c}\text { Off } \\
\text { Events } \\
N_{\text {off }}\end{array}$ & $\alpha_{\text {eff }}$ & $\begin{array}{c}\text { Excess } \\
\text { Events } \\
N_{\text {ex }}\end{array}$ & $\begin{array}{c}\text { Significance } \\
(\sigma)\end{array}$ & $\begin{array}{c}\text { Flux Upper Limit } \\
\left(10^{-12} \mathrm{~cm}^{-2} \mathrm{~s}^{-1}\right)\end{array}$ \\
\hline $0.263-0.342$ & 230 & 4726 & 0.046 & 12.6 & 0.8 & 0.5 \\
$0.342-0.445$ & 151 & 3801 & 0.046 & -23.8 & -1.9 & 0.1 \\
$0.445-0.578$ & 126 & 2905 & 0.046 & -7.6 & -0.7 & 0.2 \\
$0.578-0.751$ & 102 & 2229 & 0.046 & -0.5 & -0.1 & 0.2 \\
$0.751-0.977$ & 65 & 1663 & 0.046 & -11.5 & -1.3 & 0.1 \\
$0.977-1.269$ & 58 & 1253 & 0.046 & 0.4 & 0.0 & 0.2 \\
$1.269-1.650$ & 36 & 1033 & 0.046 & -11.5 & -1.7 & 0.1 \\
$1.650-2.145$ & 39 & 795 & 0.046 & 2.4 & 0.4 & 0.1 \\
$2.145-2.789$ & 25 & 627 & 0.046 & -3.8 & -0.7 & 0.1 \\
$2.789-3.626$ & 20 & 447 & 0.046 & -0.6 & -0.1 & 0.1 \\
$3.626-4.713$ & 14 & 354 & 0.046 & -2.3 & -0.6 & \\
\hline
\end{tabular}

Note. As for Table 2, but for selected energy ranges.

and certain data cuts (which, in this case, are the medium cuts). Above about $5 \mathrm{TeV}$, the number of events that pass the cuts is so small that the results (not shown) become very uncertain. For comparison, we also plotted the published MAGIC differential flux upper limits (Aleksić et al. 2010) in the figure, as well as the extrapolation of the best-fit power-law spectra measured with AGILE and Fermi LAT.

\subsection{Targeted Searches for TeV Gamma Rays in Radio/X-Ray States}

As mentioned in Section 1, there appears to be evidence for gamma-ray production in Cygnus X-3 only in certain radio/ $\mathrm{X}$-ray states. For a more effective search, it is, therefore, important to characterize the states that the source is in. 


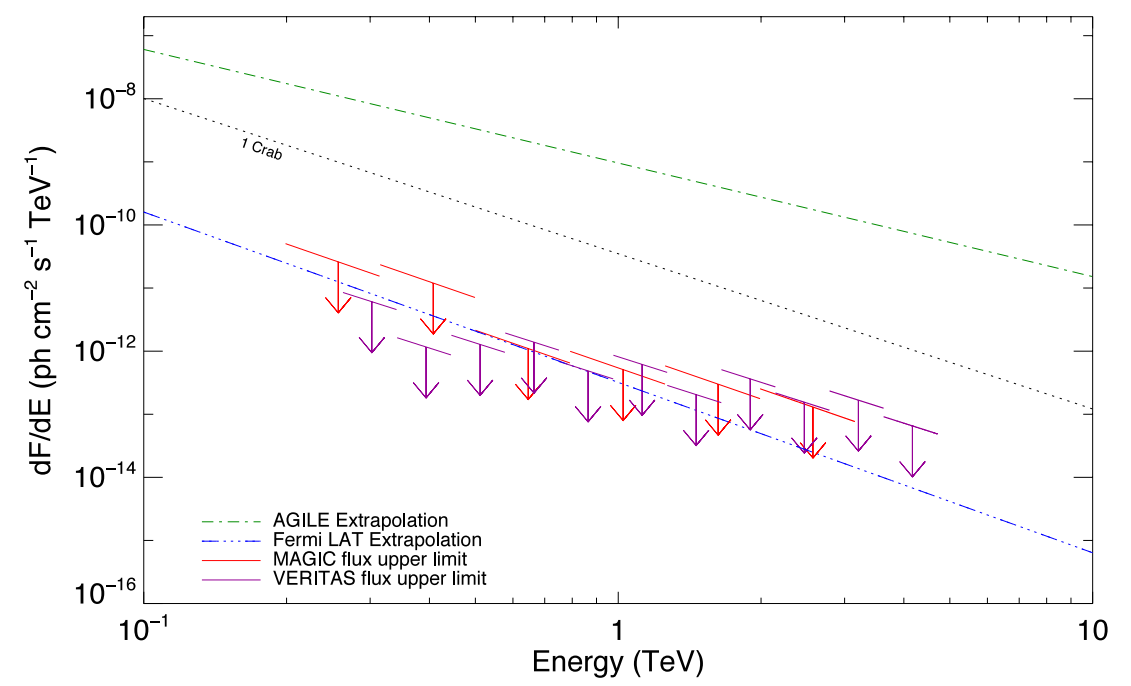

Figure 3. VERITAS 95\% C.L. differential flux upper limits of Cygnus X-3. For comparison, the published MAGIC upper limits are shown in (red) thin solid lines. See Figure 1 and Table 2 of Aleksić et al. (2010) for further details concerning the MAGIC results. A nominal spectrum of the Crab Nebula is shown in the (black) dotted line, for reference. The (blue) dot-dot-dot-dashed line and (green) dot-dashed line show the extrapolations of the power-law spectra measured with the Fermi LAT and AGILE at $\mathrm{GeV}$ energies, respectively.

(A color version of this figure is available in the online journal.)

Fortunately, there were extensive X-ray and radio coverages of Cygnus X-3 that were contemporaneous with the VERITAS observations. We used the radio and (soft and hard) X-ray light curves of the source, as shown in Figure 4, to distinguish the states, as defined in Szostek et al. (2008). We chose to divide the post-flaring state appropriately and merge it into the minor flaring and suppressed states.

It is worth noting a few key features in the multi-wavelength light curves shown in Figure 4. First, the anti-correlation between the soft and hard X-ray bands is apparent, comparing the ASM/MAXI and Swift BAT light curves. Second, the quenched state is not easily recognizable based on the radio light curve alone. It is, in fact, more apparent in the hard X-ray light curves, as it is when hard X-ray emission is quenched as well. To be more quantitative, we define the quenched state as the time when the Swift BAT flux goes below 0.01 counts $\mathrm{cm}^{-2}$ $\mathrm{s}^{-1}$ (or when the ASM flux goes above 3 counts $\mathrm{s}^{-1}$, as the soft/hard X-ray anti-correlation suggests). Finally, the times of significant detections of Cygnus X-3 at GeV energies (see the Fermi-LAT light curve) do seem to align with the transitions into or out of the quenched state (i.e., the hypersoft state) quite well.

We grouped the VERITAS observing runs based on the radio/ $\mathrm{X}$-ray states and carried out a search for $\mathrm{TeV}$ gamma rays from Cygnus X-3 for each of the states. The analysis was made with the soft, medium, and hard cuts. The results are shown separately in Table 2. No significant TeV gamma-ray signal was found in any of the searches.

\section{DISCUSSION}

The VERITAS observations of Cygnus X-3 covered the quenched state between 2008 October 30 and December 13 (MJD 54,769-54,813), when it was detected with AGILE (ATel 1827, Tavani et al. 2008b; and ATel 1848, Tavani et al. 2008a). Unfortunately, there was only one VERITAS observation in the hypersoft/quenched state on 2008 December 4 . The source was not detected at $\mathrm{TeV}$ energies. The derived flux upper limits are not very constraining (see Table 2), due to limited VERITAS exposure.
The VERITAS observations also covered the major flaring state (reaching a peak radio flux of $\sim 20 \mathrm{Jy}$ ) that followed the 2011 March quenched state. Due to the low elevation of the source and other observing constraints, VERITAS missed the peak of the radio flare (on 2011 March 24 or MJD 55,644). The source was detected during this episode with the Fermi LAT (Corbel et al. 2012). The highest LAT flux occurred on 2011 March 22, just before the peak of the radio flare. We failed to detect a signal from the source at $\mathrm{TeV}$ energies over the period from 2011 March 28 to April 5.

Based on the entire VERITAS data set, we derived, with the medium cuts, a 95\% C.L. integral flux upper limit of $0.7 \times 10^{-12}$ photons $\mathrm{cm}^{-2} \mathrm{~s}^{-1}(E>263 \mathrm{GeV})$, which is about a factor of three lower than the published MAGIC value $(E>250 \mathrm{GeV}$; Aleksić et al. 2010). Note that the difference in energy thresholds between the two measurements amounts only to an effect of a few percent. We have also made a direct comparison of the VERITAS and MAGIC constraints on differential fluxes at various energies (see Figure 3). Our upper limits are significantly lower than the MAGIC limits at lower energies. It should, however, be noted that we did not consider systematic uncertainties in our analyses, while the MAGIC results include a 30\% systematic uncertainty on flux. The VERITAS flux upper limits are compatible with the results of spectral modeling carried out by Piano et al. (2012).

If we extrapolate the best-fit Fermi LAT spectrum of Cygnus X-3 (Abdo et al. 2009) to the VERITAS energy range, following a simple power law, we would expect an integral flux of $F(E>263 \mathrm{GeV})=1.8 \times 10^{-12}$ photons $\mathrm{cm}^{-2} \mathrm{~s}^{-1}$, which is comparable to our $95 \%$ C.L. flux upper limit. However, the uncertainties on the Fermi-LAT spectrum make it difficult to conclude that a spectral break or rollover would be required from $\mathrm{GeV}$ to $\mathrm{TeV}$ energies. The episodic nature of the gamma-ray emission from Cygnus X-3 has made it even more difficult to compare Fermi LAT and VERITAS measurements. This is illustrated by the fact that the published AGILE spectrum of Cygnus X-3 (Tavani et al. 2009) is higher and harder than the Fermi-LAT spectrum. If we extrapolate the best-fit power law to the AGILE spectrum into the VERITAS energy range, we would 


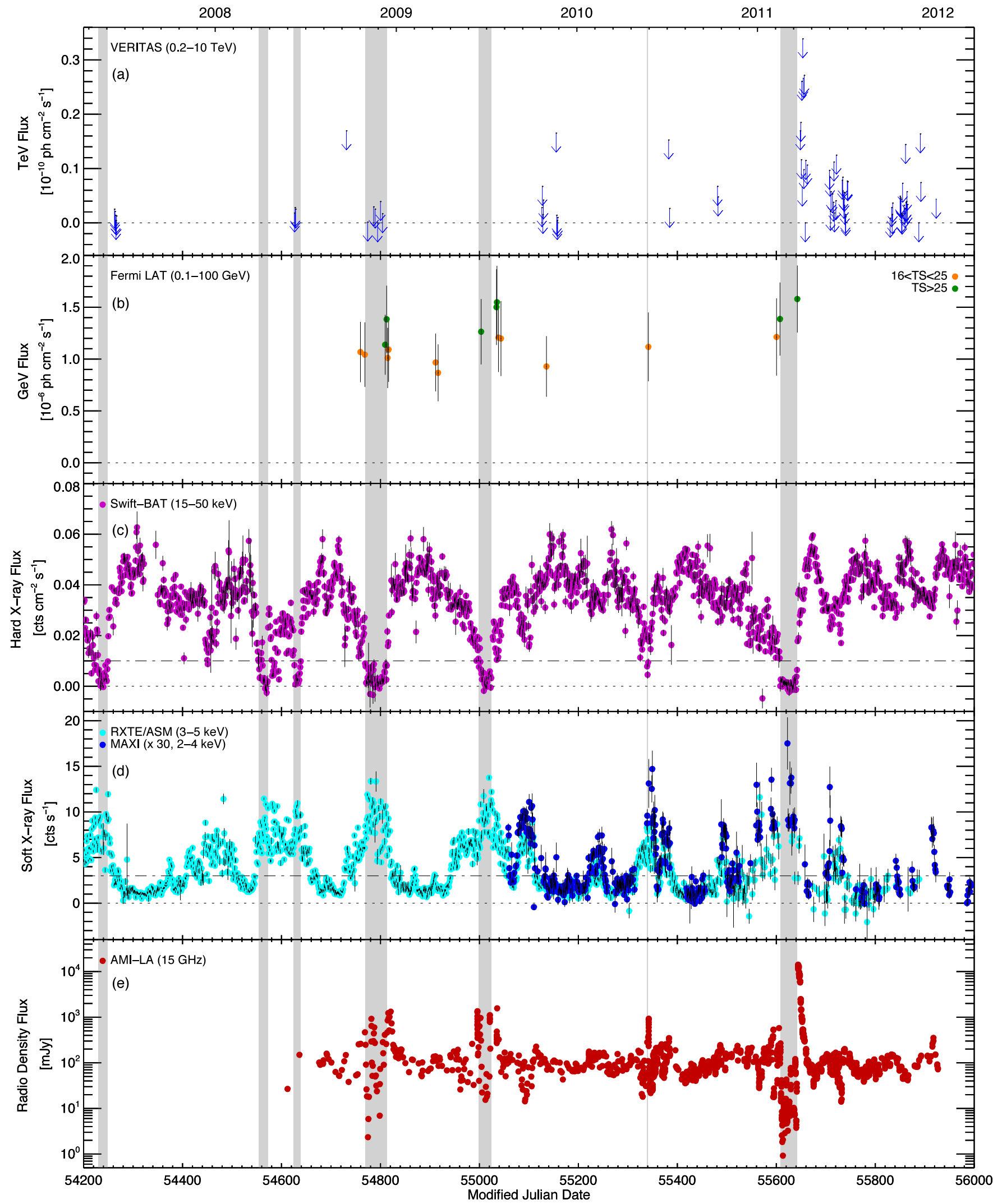

Figure 4. Multi-wavelength light curves of Cygnus X-3. Panels (a) TeV gamma ray. The VERITAS 99\% C.L. integral ( $E>263 \mathrm{GeV})$ flux upper limits are shown for individual nights. (b) $\mathrm{GeV}$ gamma ray. The data points are color-coded by the detection significance: moderate significance $(16<\mathrm{TS}<25)$ in orange, and high significance (TS > 25) in green. (c) Hard X-ray. (d) Soft X-ray. The ASM 3-5 keV measurements are shown in cyan and the MAXI 2-4 keV measurements in blue. Note that the MAXI flux values have been multiplied by 30 for clarity. (e) Radio. The AMI-LA $15 \mathrm{GHz}$ measurements are shown. The shaded areas indicate the quenched state. The dot-dashed line in (c) and (d) shows roughly the threshold for transition into or out of the quenched state.

(A color version of this figure is available in the online journal.) 
obtain an integral flux of $F(E>263 \mathrm{GeV})=3.5 \times 10^{-9}$ photons $\mathrm{cm}^{-2} \mathrm{~s}^{-1}$ (with large uncertainties). More sophisticated spectral modeling is required to connect the Fermi-LAT and VERITAS data more physically (e.g., Piano et al. 2012), but it is beyond the scope of this work.

For microquasars, the kinetic power of the jets is of the order of $\sim 10^{38} \mathrm{erg} \mathrm{s}^{-1}$, which is comparable to the bolometric luminosity of Cygnus X-3 (assuming a distance of $9 \mathrm{kpc}$; Hjalmarsdotter et al. 2008). Our flux upper limit corresponds to an upper limit on the TeV gamma-ray luminosity of $\approx 6 \times$ $10^{33} \mathrm{erg} \mathrm{s}^{-1}$. This implies a maximum gamma-ray conversion efficiency of the order $10^{-4}-10^{-5}$. In the context of leptonic models, Bednarek (2010) predicted a gamma-ray luminosity of $\approx 10^{32} \mathrm{erg} \mathrm{s}^{-1}$ for Cygnus $\mathrm{X}-3$, assuming steady emission. This is discouraging for the current generation of groundbased gamma-ray facilities, although the episodic nature of $\mathrm{GeV}$ gamma-ray emission from the source argues for more patience. In our case, the most interesting radio/X-ray state (i.e., the hypersoft state) has hardly been covered (see Table 2). A concerted, multi-wavelength effort to target this state will likely be a more effective (and resource conserving) strategy for moving forward.

This work has made use of high-level data products provided by the ASM/RXTE, MAXI, and BAT/Swift teams. The AMI-LA radio results were obtained from a public archive maintained by Guy Pooley. Paul Ray is thanked for making available the ephemeris for PSR J2032+4127. A.V. and W.C. gratefully acknowledge financial support from NASA through a Fermi GI grant (NNX11AP90G) and from Purdue University. The VERITAS operation is supported by grants from the U.S. Department of Energy Office of Science, the U.S. National Science Foundation and the Smithsonian Institution, by NSERC in Canada, by Science Foundation Ireland (SFI 10/RFP/AST2748), and by STFC in the U.K. We acknowledge the excellent work of the technical support staff at the Fred Lawrence Whipple Observatory and at the collaborating institutions in the construction and operation of the instrument.

Facilities: VERITAS, Swift, RXTE, MAXI, Fermi, AGILE, AMI

\section{REFERENCES}

Abdo, A. A., Ackermann, M., Ajello, M., et al. (Fermi LAT Collaboration) 2009, Sci, 326, 1512

Acciari, V. A., Beilicke, M., Blaylock, G., et al. (VERITAS Collaboration) 2008, ApJ, 679, 1427

Aharonian, F., Akhperjanian, A., Beilicke, M., et al. 2004, A\&A, 421, 529
Aharonian, F., Akhperjanian, A., Beilicke, M., et al. 2005, A\&A, 431, 197

Aleksić, J., Antonelli, L. A., Antoranz, P., et al. 2010, ApJ, 721, 843

Atwood, W. B., Abdo, A. A., Ackermann, M., et al. 2009, ApJ, 697, 1071

Barthelmy, S. D., Barbier, L. M., Cummings, J. R., et al. 2005, SSRv, 120, 143

Bednarek, W. 2010, MNRAS, 406, 689

Bennett, K., Bignami, G., Hermsen, W., et al. 1977, A\&A, 59, 273

Berge, D., Funk, S., \& Hinton, J. 2007, A\&A, 466, 1219

Borione, A., Chantell, M. C., Covault, C. E., et al. 1997, PhRvD, 55, 1714

Chardin, G., \& Gerbier, G. 1989, A\&A, 210, 52

Choudhury, M., Rao, A. R., Vadawale, S. V., Ishwara-Chandra, C. H., \& Jain, A. K. 2002, A\&A, 383, L35

Corbel, S., Dubus, G., Tomsick, J. A., et al. 2012, MNRAS, 421, 2947

Dubus, G., Cerutti, B., \& Henri, G. 2010, MNRAS, 404, L55

Hillas, A. M. 1985, Proc. 19th Int. Cosmic Ray Conf., ed. F. C. Jones (Washington, DC: NASA), 3, 445

Hjalmarsdotter, L., Zdziarski, A. A., Larsson, S., et al. 2008, MNRAS, 384,278

Holder, J., Aliu, E., Arlen, T., et al. 2011, Proc. 32nd Int. Cosmic Ray Conf. (Beijing), 12, 137 (arXiv:1111.1225)

Koljonen, K. I. I., Hannikainen, D. C., McCollough, M. L., Pooley, G. G., \& Trushkin, S. A. 2010, MNRAS, 406, 307

Krawczynski, H., Carter-Lewis, D. A., Duke, C., et al. 2006, APh, 25, 380

Lamb, R. C., Fichtel, C. E., Hartman, R. C., Kniffen, D. A., \& Thompson, D. J. 1977, ApJL, 212, L63

Levine, A. M., Bradt, H., Cui, W., et al. 1996, ApJL, 469, L33

Li, T. P., \& Ma, Y. Q. 1983, ApJ, 272, 317

Ling, Z., Zhang, S. N., \& Tang, S. 2009, ApJ, 695, 1111

Matsuoka, M., Kawasaki, K., Ueno, S., et al. 2009, PASJ, 61, 999

Mattox, J. R., Bertsch, D. L., Chiang, J., et al. 1996, ApJ, 461, 396

McCollough, M. L., Robinson, C. R., Zhang, S. N., et al. 1999, ApJ, 517, 951

Miller-Jones, J. C. A., Blundell, K. M., Rupen, M. P., et al. 2004, ApJ, 600,368

Mioduszewski, A. J., Rupen, M. P., Hjellming, R. M., Pooley, G. G., \& Waltman, E. B. 2001, ApJ, 553, 766

Mori, M., Bertsch, D. L., Dingus, B. L., et al. 1997, ApJ, 476, 842

Nolan, P. L., Abdo, A. A., Ackermann, M., et al. 2012, ApJS, 199, 31

O’Flaherty, K. S., Cawley, M. F., Fegan, D. J., et al. 1992, ApJ, 396, 674

Ong, R. A. 1998, PhR, 305, 93

Piano, G., Tavani, M., Vittorini, V., et al. 2012, A\&A, 545, A110

Predehl, P., Burwitz, V., Paerels, F., \& Trümper, J. 2000, A\&A, 357, L25

Ray, P. S., Kerr, M., Parent, D., et al. 2011, ApJS, 194, 17

Rolke, W. A., López, A. M., \& Conrad, J. 2005, NIMPA, 551, 493

Romero, G. E., Torres, D. F., Kaufman Bernadó, M. M., \& Mirabel, I. F. 2003, A\&A, 410, L1

Schilling, M., Mang, O., \& Siems, M. 2001, Proc. 27th Int. Cosmic Ray Cong. (Hamburg), 6, 2521

Szostek, A., Zdziarski, A. A., \& McCollough, M. L. 2008, MNRAS, 388, 1001

Tavani, M., Bulgarelli, A., Piano, G., et al. 2009, Natur, 462, 620

Tavani, M., Piano, G., Chen, A., et al. 2008a, ATel, 1848, 1

Tavani, M., Sabatini, S., Piano, G., et al. 2008b, ATel, 1827, 1

van Kerkwijk, M. H., Geballe, T. R., King, D. L., van der Klis, M., \& van Paradijs, J. 1996, A\&A, 314, 521

Waltman, E. B., Fiedler, R. L., Johnston, K. L., \& Ghigo, F. D. 1994, AJ 108,179

Weekes, T. C. 1992, SSRv, 59, 315

Zdziarski, A. A., Maitra, C., Frankowski, A., Skinner, G. K., \& Misra, R. 2012, MNRAS, 426, 1031

Zwart, J. T. L., Barker, R. W., Biddulph, P., et al. 2008, MNRAS, 391, 1545 